\title{
A SURVEY ON EXTENSIONS OF THE PURE GRAVITY SWING-BY MANEUVER
}

\author{
A. F. S. Ferreira ${ }^{1}$, R. V. de Moraes $^{1,2}$, A. F. B. A. $\operatorname{Prado}^{2,3}$, O. C. Winter ${ }^{1}$, and V. M. Gomes ${ }^{1}$ \\ Received March 30 2021; accepted August 132021
}

\begin{abstract}
The present paper surveys the more recent techniques related to the swing-by maneuver, where a spacecraft changes its energy and angular momentum by passing close to celestial bodies. It is focused on the literature related to extensions of this maneuver, with emphasis in the powered version, where an impulse is applied to the spacecraft near the closest approach. Several mathematical models are considered, including the patched-conics approximation for analytical studies, and the restricted three-body problem for the numerical simulations. The main goal is to show the models and the main conclusions available in the literature for those maneuvers. Some key results are shown to discuss important aspects of this maneuver, including the analysis of the energy variation of the spacecraft, the behavior of the trajectories and other applications.
\end{abstract}

\section{RESUMEN}

Presentamos una reseña de los resultados recientes sobre la maniobra de columpio, en la cual una nave espacial cambia su energía y momento angular al pasar cerca de un cuerpo celeste. Nos concentramos en la literatura sobre las extensiones de esta maniobra, con énfasis en la versión con potencia aplicada, en la cual se le impone a la nave un impulso durante el máximo acercamiento. Se consideran varios modelos matemáticos, incluyendo la aproximación de la cónica parcial para estudios analíticos y el problema restringido de los tres cuerpos para las simulaciones numéricas. Nuestro objetivo principal es mostrar los modelos y las conclusiones disponibles en la literatura para estas maniobras. Con los principales resultados discutimos aspectos interesantes de la maniobra, incluyendo el análisis de la variación de la energía de la nave y el comportamiento de las trayectorias.

Key Words: celestial mechanics — methods: numerical — minor planets, asteroids: general - space vehicles - surveys

\section{INTRODUCTION}

Missions to distant planets, asteroids and comets have been planned and performed frequently by space agencies around the world. A significant and important issue for the development of these missions is the high fuel consumption required by those missions. It is well known that the cost to send a spacecraft to space is high and one of the most expensive parts of the mission is the large amount of fuel necessary to send materials to space. Therefore, to minimize this cost is a fundamental prob-

\footnotetext{
${ }^{1}$ São Paulo State University - FEG-UNESP, Brazil.

${ }^{2}$ National Institute for Space Research - INPE, Brazil.

${ }^{3}$ University of Russia - RUDN University, Russia.
}

lem in astrodynamics. The well-known bi-impulsive Hohmann transfer (Hohmann 1925) is a major and first step into this problem, studying the minimum fuel consumption to transfer a spacecraft between two co-planar circular orbits. This maneuver shows that a transfer ellipse tangential to both the initial and final orbits is the solution that gives the minimum increments of velocities. After that, Hoelker \& Silber (1959) and Shternfeld (1959) showed that the tri-impulsive bi-elliptic transfer is more economical to transfer a spacecraft between two coplanar circular orbits, if the radius of the external orbit divided by the radius of the interior orbit is larger than 11.93875. Those are the first solutions to the prob- 
lem of minimizing fuel consumption in space maneuvers, which will later include gravity assisted maneuvers.

After that, several, alternatives of maneuvers with minimum fuel consumption appeared in the literature, showing extensions of those two important bi-impulsive transfers. Some examples are found in Edelbaum (1959), Roth (1965), Jezewski \& Rozendaal (1968), Jin \& Melton (1991) and Prussing (1992). To reduce even more the fuel expenditure, it is also possible to consider low thrusts, where a small force is applied to the spacecraft for a relatively long time. The advantage of this approach is the high specific impulses of those thrusts, which can generate large increments of velocity with a small mass of fuel consumed. Good examples are shown in Lawden (1953), Lawden (1954), Sukhanov \& Prado (2001), Casalino \& Colasurdo (2007), Ozimek \& Howell (2010), Zhang et al. (2015), Carvalho et al. (2016) and Fernandes \& Carvalho (2018). A particularly interesting example is shown in Qi \& Ruiter (2020). A method to obtain preliminary continuous thrust powered swing-by (PSB) orbits was constructed considering a series of discrete optimal single impulsive PSBs. Using these initial guesses, optimal continuous thrust PSB orbits were designed by using optimal control problem techniques. Another good example is available in Konstantinov \& Thein (2017), which simplifies the problem using a method which allows us to avoid the difficulties of solving the optimization problem of the interplanetary trajectory of a spacecraft propelled by electric power using a sequence of swing-bys.

Considering those advances in the swing-by techniques, the present paper has the goal of making a complete survey, covering all the options available for mission designers. Some of the main models are briefly explained and the pertinent literature is indicated for more details. Particular importance is given to the powered version of this maneuver, where an impulse is combined with the close approach. The different mathematical models used to solve this problem are also showed and key results are discussed, in particular in terms of the energy variations of the spacecraft. Several important applications are described, also indicating the appropriate literature.

The following sections describe the history and mathematics behind the pure gravity swing-by maneuver, when only the gravity of the body is considered in maneuver, and the powered swing-by (PSB), when propulsion is applied to the probe to improve the maneuver. In $\S 2$, the history and applications of the pure gravity maneuver in space missions are presented, as well as an introduction on the extension of the pure gravity maneuver to the powered swingby maneuver. $\S 3$ shows the mathematical model of the pure maneuver, including the "patched conics approach" for an analytical study and the "circular and elliptical restricted three-body problem" for numerical simulations, in addition to the analytical equations that show the effect of the maneuver. Throughout the work the historical context is cited. $\S 4$ presents the different types of powered extensions available in the literature. $\S 5$ shows a detailed description of the powered swing-by maneuver, focusing on the mathematical and geometric presentation of the problem, in addition to the historical description, and presenting a summary of the analyzes available in the literature. Finally, $\S 6$ talks about captures of the spacecraft.

\section{THE PURE GRAVITY SWING-BY MANEUVER}

To reduce even more the fuel consumption in orbital maneuvers, a new concept appeared in the literature of space missions, the swing-by maneuver. Negri \& Prado (2020) show a historical description of the early years of this maneuver, considering the pure gravity maneuver. In this sense, the present paper is a continuation of Negri \& Prado (2020), showing some of the later advances and generalizations of this technique, covering all the options available for mission designers. Particular importance is given, in this work, to the powered version of this maneuver, where an impulse is combined with the close approach. Some of the main models are briefly explained and the pertinent literature is indicated for more details.

In terms of practical applications, Minovitch, in 1961, wrote an important document (Minovitch 1961), where he showed the basic physics involved in this close approach maneuver when applied to spacecraft trajectories, and the possibility of using this technique to send a spacecraft out of the Solar System with a much smaller fuel consumption, when compared to maneuvers made exclusively based in propulsion systems. A detailed description of the research developed by Minovitch is shown in Dowling et al. (1990) and Dowling et al. (1991). Looking at real missions that already used this technique, it is possible to find several examples. Flandro (1966) showed a detailed plan for the Voyager mission, which was composed by two spacecraft that made a series of close approaches to several planets of the 
Solar System to gain energy for the trip. The calculations were made based on the equations developed by Minovitch (1961), and it was possible to verify the efficiency of this maneuver in a grand tour of the Solar System. It was one of the most important interplanetary missions that would not have been possible without the use of close approaches to the planets visited. Several other missions used passages by the planets of the Solar System to increase their energy, so helping the spacecraft to complete the goals of the mission. Some examples are shown in Farquhar \& Dunham (1981) and Farquhar et al. (1985). Another good example is the Galileo mission, which used pure gravity swing-by maneuvers to gain energy, as shown in D'Amario et al. (1981), D'Amario et al. (1982) and Byrnes \& D'Amario (1982). This same concept was also used in the Messenger and Bepi-Colombo missions, which were two missions directed to the planet Mercury (McNutt et al. 2004, 2006; Grard 2006), but in this case the goal was to reduce the two-body energy Sun-spacecraft. Those possibilities will be explained later in the present paper.

One of the good descriptions of this maneuver available in the literature is shown in Broucke (1988). It is a paper presenting the basic celestial mechanics of the pure gravity assisted maneuver. A few books also mention this problem, but usually very quickly, like Ruppe (1967), Opik (1976) and Kaplan (1976).

Looking for extensions of this pure gravity based maneuver, the concept of "powered swing-by maneuver" appeared in the literature, which is a combination of a pure gravity swing-by, which depends only on the gravity field of the celestial body approached and the geometry of the passage, with the application of an impulse in the spacecraft at some point inside the sphere of influence of the body involved in the maneuver. The use of this maneuver is an important option when the energy obtained from the pure gravity swing-by maneuver is not enough to complete a given mission. It gives new options to the mission design and can enable missions that could not be achieved without this technique. The best choice for the geometry can give higher energy variations to the spacecraft and, consequently, more velocity after it leaves the system. If the objective is to remove energy to insert the spacecraft in orbit around a celestial body, this type of maneuver is also efficient. There are studies that analyze the best geometry for this maneuver and the effects of the parameters related to it, which are described in some detail later. When considering elliptical systems (which increases the accuracy of the results, in particular when the planets Mars and Mercury are involved, because they have larger eccentricities) it is necessary to use two more parameters to describe the maneuver, both of them related to the geometry of the primaries: the eccentricity of their orbits and the true anomaly of the secondary body in its orbit around the primary at the moment of closest approach.

To show that the powered swing-by maneuver can benefit some missions, it is necessary to make a detailed comparison between maneuvers performed with the impulse applied near the close approach of the spacecraft around the celestial body and maneuvers performed with the impulse applied after the spacecraft leaves the sphere of influence of the body. This point is shown in Striepe \& Braun (1991) in a mission from Earth to Mars making a powered swing-by in Venus. Following that, Prado (1996) made a detailed and general analysis of this maneuver for circular systems, to verify its efficiency. The author considered the situation where the impulse is applied to the spacecraft at the moment when it passes through the periapsis of its orbit, as well as the situation where the impulse is applied at a point outside the sphere of influence of the secondary body. The results showed that, in many circumstances, to apply the impulse at the periapsis has advantages in terms of getting extra gains of energy from the impulsive maneuver. This study was followed by Casalino et al. (1999a), which extended this maneuver for the situation where the application of the impulse is not made at the periapsis of the orbit of the secondary body. The next step was to consider this problem when the impulse is applied in the periapsis of the close approach trajectory in systems with elliptical orbits for the primaries, thus studying the effects of the eccentricity of the primaries on the maneuver. It was done in Prado (1997), Ferreira et al. (2017) and Ferreira et al. (2018a). It is important to study those systems, because elliptical systems are numerous in space, and important celestial bodies, like the planets Mars and Mercury, are among them, and they have been used for swing-by maneuvers. The results showed that the eccentricity gives strong effects in the maneuver.

Taking into account those considerations, this review has the goal of presenting a more detailed discussion of some of the main extensions of the swingby maneuver, including the key conclusions available in the literature. Before going in detail into this maneuver, an introduction to the work related to the unpowered version of the maneuver is also made, to explain the most important aspects of this technique 
and some lines of research related to them. The reader interested in more details about this history should look at Negri \& Prado (2020).

\section{MATHEMATICAL MODELS FOR THE PURE GRAVITY SWING-BY MANEUVERS}

Analytical and numerical studies are available in the literature regarding the pure gravity swing-by maneuver, and both approaches will be summarized next. Analytical studies are based on the "patchedconics" model and numerical studies derive from the use of the restricted three-body problem (RTBP) for the circular and elliptic case (Szebehely 1967).

\section{1. "Patched-Conics" Approach}

The "patched-conics" approach (Bate et al. 1971) is a method for simplifying the calculation of trajectories that employs the concept of the sphere of influence of a celestial body. The approximation is made by dividing the space into several parts and then assigning a sphere of influence to each body of the system.

When the spacecraft is within the sphere of influence of a body, only the effect of the gravitational force of this body is considered, regardless of the size of the bodies. The effects of the forces coming from the other bodies of the system are neglected and the spacecraft follows a Keplerian orbit. For a swingby maneuver the sequence is the following (Broucke 1988):

- The spacecraft $\left(M_{3}\right)$ initially comes from an orbit around the main body of the system $\left(M_{1}\right)$. At this phase of the motion only the gravitational force of $M_{1}$ acts on $M_{3}$, and the effect of the gravity of the secondary body $\left(M_{2}\right)$ is neglected;

- When $M_{3}$ reaches a certain distance from $M_{2}$, its gravity starts to dominate the motion of the spacecraft, and the effect of $M_{1}$ can be neglected, so the gravity of the secondary body $\left(M_{2}\right)$ is assumed to be the only force acting in the motion of $M_{3}$;

- Then, the spacecraft escapes from $M_{2}$ and goes again toward the main body. The gravity of $M_{1}$ is then assumed to be the only force acting in the spacecraft again.

This method reduces the more complex threebody problem to a sequence of two-body problems, for which the solutions are the usual conic sections (Keplerian orbits).

\subsection{Circular and Elliptical Restricted Three Body Problem}

The "three-body problem" is the simplest particular case, and perhaps the most popular one, of the Nbody problem. It is a non-integrable problem so, in general, there is no analytical solution, but for some mass values and initial conditions there are periodic or quasi-periodic solutions. This is the case of the "restricted three-body problem", that can be circular or elliptical. In this version, there is a larger body, called $M_{1}$, which is the primary body of the system. It is followed by the secondary body $M_{2}$, the second largest in mass, and the problem studies the motion of a massless body $M_{3}$, which can be a spacecraft that orbits the system formed by the other two bodies.

The circular restricted three-body problem (CRTBP) (Szebehely 1967; Murray \& Dermott 2000; Valtonen \& Karttunen 2006; Pourtakdoust \& Sayanjali 2014; Zotos 2015; Qian et al. 2016) assumes that $M_{1}$ and $M_{2}$ are in circular orbits around the common center of mass of the system. In this review, the motion is always limited to the orbital plane of the primaries. The mass of $M_{1}$ is given by $1-\mu$, with $\mu=\frac{m_{2}}{\left(m_{1}+m_{2}\right)}$ the mass parameter of the system. $m_{1}$ and $m_{2}$ are the masses of the bodies $M_{1}$ and $M_{2}$, respectively. These choices are compatible with the canonical system of units, which is a system where the total mass of the system is one; the unit of distances is the semi-major axis $(a)$ of the orbit of the primaries; the gravitational constant is one; and the unit of time is defined such that the orbital period of the primaries is $2 \pi$ (Szebehely 1967).

For the elliptical restricted three-body problem (ERTBP), the bodies $M_{1}$ and $M_{2}$ are moving in elliptical orbits around their common center of mass (Szebehely 1967; Murray \& Dermott 2000; Valtonen \& Karttunen 2006; Szebehely \& Giacaglia 1964). From basic celestial mechanics, it is known that the distance between $M_{1}$ and $M_{2}$ is given by $d=\frac{a\left(1-e^{2}\right)}{(1+e \cos \nu)}$, where $a$ is the semi-major axis, $e$ is the eccentricity of the orbit of the primaries and $\nu$ is the true anomaly of the secondary body. The equations of motion (equation 1) are integrated in time and the mathematical model can be built using a reference system with the origin fixed in the center of mass of the bodies

$$
\begin{aligned}
& \ddot{x}=-\frac{(1-\mu)\left(x-x_{1}\right)}{r_{1}^{3}}-\frac{\mu\left(x-x_{2}\right)}{r_{2}^{3}}, \\
& \ddot{y}=-\frac{(1-\mu)\left(y-y_{1}\right)}{r_{1}^{3}}-\frac{\mu\left(y-y_{2}\right)}{r_{2}^{3}} .
\end{aligned}
$$




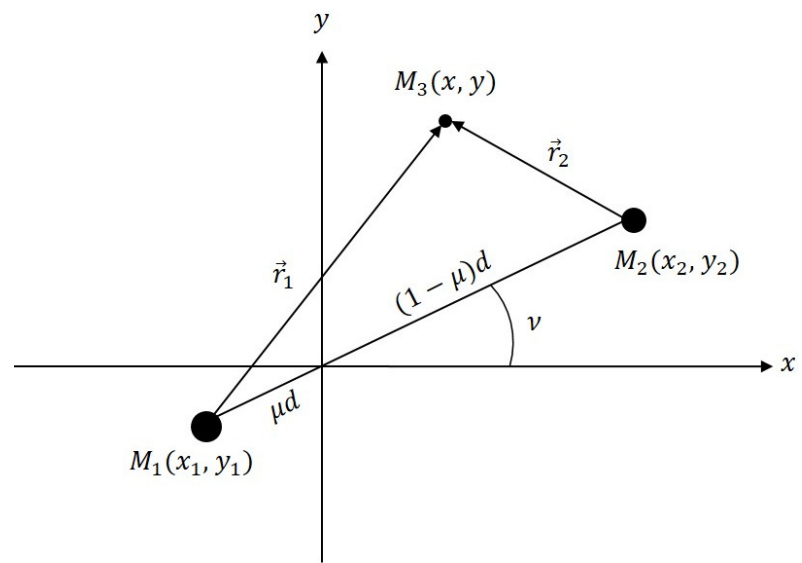

Fig. 1. Geometry of the restricted three-body problem.

In those equations, $r_{1}=\sqrt{\left(x-x_{1}\right)^{2}+\left(y-y_{1}\right)^{2}}$ represents the distance between $M_{1}$ and the spacecraft; $r_{2}=\sqrt{\left(x-x_{2}\right)^{2}+\left(y-y_{2}\right)^{2}}$ the distance between $M_{2}$ and the spacecraft; $(x, y)$ the position of the spacecraft; $\left(x_{1}, y_{1}\right)$ and $\left(x_{2}, y_{2}\right)$ the positions of $M_{1}$ and $M_{2}$, respectively, as shown in Figure 1.

In contrast to the patched-conics model, simulations in the CRTBP and ERTBP consider the influence of the two massive bodies on the spacecraft at all times. There are no analytical solutions, but the numerical approach generates more accurate results than the "patched conics" approach.

\subsection{Analytical Equations to Measure the Effects of the Pure Gravity Swing-by Maneuver}

After reviewing those basic concepts, it is time to look in more detail at the pure gravity swing-by maneuver. Broucke (1988) presented a theoretical exposition of the celestial mechanics and the fundamental principles of the gravity-assisted maneuver, or pure gravity swing-by. It started from references that investigated this maneuver before, like Deerwester (1966); Hiller (1969); Longman (1970); Hollenbeck (1975); Diehl \& Myers (1987) and Diehl et al. (1987). The most common goal of this maneuver is to give energy to the spacecraft to follow its journey, but there are also some researches showing maneuvers ending in captures by the secondary body of the system after a close approach (Helton 1972; Cline 1979; Nock \& Upholf 1979).

Broucke (1988) derived analytically, from the patched-conics approach, the geometric results of the maneuver, which was basically obtained from the rotation of the velocity vector of the spacecraft at the time of close approach. He also calculated the variations in energy and angular momentum of the space-

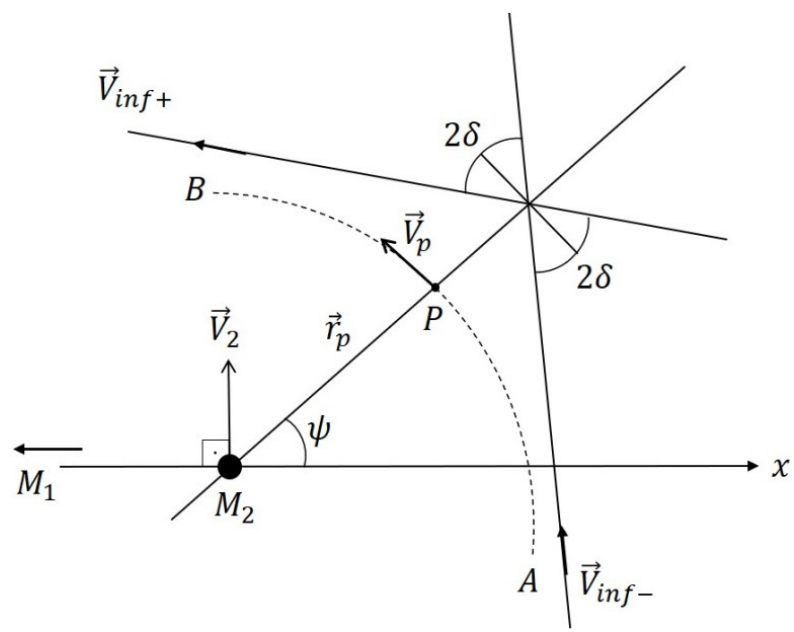

Fig. 2. Geometry of the pure gravity swing-by (adapted from Broucke (1988)).

craft with respect to the primary body of the system. Besides that, he made numerical integrations based on the restricted three-body problem, to get more accurate trajectories.

The maneuver is realized around the less massive primary of the system and the variations are measured with respect to the main body of the system. The system has circular orbits, angular velocity $\omega$ and planar motion.

Figure 2 is an adaptation based on Broucke (1988). The spacecraft comes from a Keplerian twobody orbit with main body $\left(M_{1}\right)$, passes close to the secondary body $\left(M_{2}\right)$, and is affected by the gravity of this body, which causes a rotation of the velocity vector of the spacecraft. Then, it moves away from the secondary body to a new Keplerian two-body orbit around $M_{1}$. The angle of curvature of the velocity vector is $2 \delta$ for the whole maneuver; $\vec{V}_{i n f-}$ is the approach velocity of the spacecraft relative to $M_{2} ; \vec{V}_{i n f+}$ is the escape velocity relative to $M_{2} ; \psi$ is the angle of approach; $\vec{r}_{p}$ is the radius of the periapsis and $\vec{V}_{2}$ the velocity of $M_{2}$ around the center of mass of the system, which makes an angle of $90 \mathrm{deg}$ with the $x$-axis. From this geometry Broucke derived equations 2-4 for the variations of velocity, energy and angular momentum of the pure gravity maneuver

$$
\begin{gathered}
\Delta V=2 V_{i n f-} \sin \delta, \\
\Delta E=-2 V_{2} V_{i n f-} \sin \delta \sin \psi, \\
\Delta C=-2 \omega V_{2} V_{i n f-} \sin \delta \sin \psi .
\end{gathered}
$$


Examining the formula for $\Delta E$, an analysis of the configuration of the gains and losses of energy due to the maneuver can be made (Broucke 1988). If $0<\psi<180 \mathrm{deg}$, the periapsis of the orbit of the spacecraft is in front of $M_{2}$ and there are losses of energy due to gravity. Missions like Mariner 10, Messenger and BepiColombo (McNutt et al. 2004, 2006; Grard 2006; NASA 1999-2012, 2021; Dunne \& Burgess 1978; Jehn et al. 2008) used the pure gravity swing-by to remove energy from the spacecraft to insert it into orbit. On the opposite side, if $180<\psi<360 \mathrm{deg}$, the periapsis of the orbit of the spacecraft is behind $M_{2}$ and the spacecraft gains energy due to the gravity of the secondary body. Broucke (1988) also included a classification of the types of orbits resulting from the gravity assisted maneuver, obtained from numerical explorations of the problem using the restricted three-body problem.

An analytical equation to show the variation of energy of the spacecraft in a pure gravity elliptic swing-by maneuver was derived in Ferreira et al. (2018b). It is shown in equation 5 below. It extends the literature by expanding analytical studies based in the patched-conics approach to elliptical orbits. In this equation, " $a$ " is the semi-major axis of the orbit of the primaries, " $e$ " is the eccentricity of this orbit, $\mu$ the mass parameter of the system, $V_{i n f}$ the velocity of approach of the spacecraft when getting closer to $M_{2}, \nu$ the true anomaly of $M_{2}$ when the close approach occurs, $\psi$ is the angle of approach and $\beta=\cos ^{-1}\left(-\frac{V_{r}}{V_{2}}\right)$, with $V_{r}$ the radial velocity of $M_{2}$, and $V_{2}$ the magnitude of the velocity of $M_{2}$ with respect to $M_{1}$

$\Delta E=2 V_{i n f} \sqrt{(1-\mu)\left(\frac{2}{\frac{a\left(1-e^{2}\right)}{1+e \cos \nu}}-\frac{1}{a}\right)} \sin \delta \cos (\psi+\beta)$.

Equation 5 shows that the energy variation is dependent on the angle between the velocity of $M_{2}$ around the center of mass and the axis connecting the primaries $\left(180^{\circ}-\beta\right)$. The angular momentum variation is also obtained (equation 6 ), and it is shown that it is directly dependent on the distance between the bodies $d=\frac{a\left(1-e^{2}\right)}{(1+e \cos \nu)}$, but not on the angle $\beta$

$$
\Delta C=-2 \frac{a\left(1-e^{2}\right)}{1+e \cos \nu} V_{i n f} \sin \delta \sin \psi .
$$

This same reference (Ferreira et al. 2018b) identified the configurations of maximum gains and losses of energy due to the gravity effect of the secondary body. It concluded that maneuvers with energy loss occur when $90<\psi+\beta<270$ deg, with maximum loss at $\psi+\beta=180 \mathrm{deg}$. The region of gains of energy is $-90<\psi+\beta<90 \mathrm{deg}$, with maximum in $\psi+\beta=0 \mathrm{deg}$ or $\psi+\beta=360 \mathrm{deg}$. The effect of the swing-by in the energy variation is zero when $\psi+\beta=90$ deg or $\psi+\beta=270$ deg. Numerical simulations validated the equations presented in the paper. It was also shown that the variation of velocity in the elliptic maneuver does not depend on the orbit of the secondary body. It remains the same as that obtained from the circular maneuver.

Regarding differences in the results obtained from the two-and restricted three-body dynamics in the pure gravity maneuver, there are also some comparisons available in the literature measuring the effects of the analytical approximations on the results for a swing-by maneuver. Good examples are Campagnola et al. (2012); Negri et al. (2017) and Negri et al. (2019), which show the errors made by the patched-conics approach in different aspects, including those errors as a function of the mass parameter of the system.

Several other applications of this type of maneuver exist, like the use of the inner planets of the Solar System to send a spacecraft to the Sun, considered in Sukhanov (1999); or a mission leaving and returning to the Earth with an intermediate swing-by with the Moon (Gagg Filho \& Fernandes 2018). A graphical method to solve this problem is developed in Strange \& Longuski (2002). Some references also studied the problem of multiple swing-by maneuvers, like (Dunham \& Davis 1985; Marsh \& Howell 1988; Ross \& Scheeres 2007; Jerg et al. 2009; Vasile \& Campagnola 2009; Kloster et al. 2011). Swing-by with a cloud of particles was also studied, like in Gomes \& Prado (2008, 2010); Gomes et al. (2013); Formiga et al. (2018). Other studies showed the flyby transfers between Sun Earth Moon libration point orbits with different energies in the Sun Earth Moon restricted four-body problem, like in Qi et al. (2017). There are also some researches considering the particular case of orbits for a spacecraft travelling around the Sun and perturbed by Jupiter, even when the spacecraft does not enter the sphere of influence of the planet (Carusi et al. 1982; Greenberg et al. 1988; Carusi et al. 1990, 1995).

Swing-bys are also considered in the "endgame problem", whose goal is to minimize the energy required to insert a spacecraft around a celestial body at the end of a multi-body mission (Woolley \& Scheeres 2009; Campagnola \& Russell 2010a,b). A dynamical system approach, using the RTBP as the mathematical model, is also used to study the pure 
gravity swing-by maneuvers in Koon et al. (2000, 2001, 2002, 2008); Howell et al. (2001); Anderson (2005); Belbruno et al. (2008); Anderson \& Lo (2010, 2011) and Qi \& Ruiter (2018a).

\section{EXTENSIONS OF THE PURE GRAVITY MANEUVER}

Another version of the family of the close approach maneuver available in the literature is the one where the atmosphere of a planet is used to change the trajectory of the spacecraft, which is a type of transition to the powered maneuver, since the atmosphere acts like a continuous propulsion applied to the spacecraft during the atmospheric flight. It is the so called "aero-gravity assisted maneuver". Several papers exist covering this topic. Some of them are more generic, trying to understand the whole effect of the atmosphere and the shape of the spacecraft, like McRonald \& Randolph (1992); Lohar et al. (1994); Elices (1995); Lohar (1996); Bonfiglio et al. (2000); Armellin et al. $(2006,2007)$ and Prado \& Broucke (1995). There are also studies concentrated on the atmosphere of the Earth, which has more accurate models available for its density distribution, like Gomes et al. $(2013,2016)$ and Piñeros \& Prado (2017). Another main line of research includes atmospheres of planets and focuses on interplanetary trajectories using the atmosphere of an intermediate planet, like in Gillespie \& Ross (1967); Lewis \& McRonald (1992); Randolph \& McRonald (1992); Sims et al. (1995, 2000); Lohar et al. (1997); Lavagna et al. (2005); Murcia et al. (2018a,b).

The literature also considers space maneuvers that include a combination of low thrust and a close approach by a celestial body, in two separate steps. Examples are shown in Casalino et al. (1999b); McConaghy et al. (2003) and Okutsu et al. (2016).

Applications of the gravity-assisted maneuvers also exist linked to the gravitational capture maneuver (Belbruno 1987, 1990, 1994; Yamakawa 1992; Yamakawa et al. 1992, 1993; Belbruno \& Miller 1993; Machuy et al. 2007), where one or more swing-bys are performed to help the capture of a spacecraft by a celestial body.

\section{THE POWERED SWING-BY MANEUVER}

As already explained, the powered swing-by maneuver is a technique where the spacecraft approaches a celestial body and uses the gravity of this body to modify its trajectory, but the maneuver is combined with an impulse applied to the spacecraft by a propulsive system. It is a more complex maneuver when compared to the pure gravity "swing-by" maneuver, where the only forces acting in the motion of the spacecraft are the gravity fields of the celestial bodies involved. The impulse can be applied in different locations of the trajectory of the spacecraft and in different directions, but always inside the sphere of influence of the celestial body. In this way, the main objective of these researches is to study the effects of different geometries and characteristics of the impulse in the variations of the energy of the spacecraft, in particular in finding the locations of the points and directions of the impulses that gives maximum and minimum variations of energy.

This particular maneuver is also an extension of the pure gravity maneuver, and the main goal of the present paper is to review and to describe it in more detail. The study made by Broucke (1988) was later expanded by adding an impulse to the spacecraft during the maneuver, at the time of the close approach (Prado 1996). This impulse is free to vary its magnitude and direction. A set of analytical equations is derived which calculate the increment in velocity, energy and angular momentum of the spacecraft after the combined maneuver, similarly to the ones available in Broucke (1988), but with the presence of the impulse (Ferreira et al. 2018a). The most important ones are shown in equations (7-10), which give the variations of velocity and energy.

The velocity variation is given by $\Delta \vec{V}=\vec{V}_{o}-\vec{V}_{i}$, being $\vec{V}_{i}=\vec{V}_{i n f-}+\vec{V}_{2}$ the approach velocity and $\vec{V}_{o}=\vec{V}_{i n f+}+\vec{V}_{2}$ the departure velocity of the spacecraft, both with respect to $M_{1}$. Soon, $\Delta V=\sqrt{\left(V_{i n f+x}+V_{i n f-x}\right)^{2}+\left(V_{i n f+y}+V_{i n f-y}\right)^{2}}$, obtained from the components of $\vec{V}_{i n f-}$ and $\vec{V}_{i n f+}$, which are given by equations 7 .

$$
\begin{aligned}
& V_{i n f-x}=-V_{i n f-} \sin (\psi-\delta), \\
& V_{i n f-y}=V_{i n f-} \cos (\psi-\delta), \\
& V_{i n f+x}=-V_{i n f+} \sin (\psi-\delta+\Theta), \\
& V_{i n f+y}=V_{i n f+} \cos (\psi-\delta+\Theta),
\end{aligned}
$$

where $\Theta=\delta-f_{0}+f_{\text {lim }}+90$ deg is the total deflection angle of the impulsive maneuver derived in Ferreira et al. (2018a); $f_{0}$ is the angle between the radius of the periapsis $\left(\vec{r}_{p}\right)$ of the initial maneuver and the new radius of the periapsis $\left(\vec{r}_{p+}\right)$ obtained after the application of the impulse; $f_{\text {lim }}$ is the true anomaly of the asymptotes of the second orbit of the spacecraft, after the impulse is applied; and the other variables have already been defined in Figure $1, \S 3$.

$$
\Delta V=\sqrt{V_{i n f-}^{2}+V_{i n f+}^{2}-2 V_{i n f-} V_{i n f+} \cos \Theta} .
$$


For the energy variation we have $\Delta E=\frac{1}{2}\left(\vec{V}_{o}^{2}-\vec{V}_{i}^{2}\right)$, with $\vec{V}_{o}$ and $\vec{V}_{i}$ being already defined, and the components of $\vec{V}_{2}$ given by $\vec{V}_{2}=\left(-V_{2} \cos \beta, V_{2} \sin \beta\right)$, where $180 \operatorname{deg}-\beta$ is the angle between $\vec{V}_{2}$ and the $M_{1}-M_{2}$ line. The complete mathematical and geometric explanation of these equations is given in Ferreira et al. (2018a).

$$
\begin{aligned}
\Delta E= & \frac{1}{2}\left(V_{i n f+}^{2}-V_{i n f-}^{2}-2 V_{2} V_{i n f-} \sin (\beta-\delta+\psi)+\right. \\
& \left.2 V_{2} V_{i n f+} \sin (\beta-\delta+\Theta+\psi)\right) .
\end{aligned}
$$

This paper also shows the efficiency of the powered swing-by maneuver, which is defined as the amount of extra energy that is obtained by the spacecraft when the impulse is applied at the moment of closest approach, if compared to a maneuver where an impulse, with the same magnitude, is applied after the spacecraft leaves the sphere of influence of $M_{2}$. The analysis was performed according to the gravitational parameter of the secondary body $(\mu)$, periapsis radius $\left(r_{p}\right)$, orientation of the swing-by maneuver $(\psi)$ and approach velocity $\left(V_{i n f-}\right)$. The reason for the positive efficiency of this maneuver is that when the impulse is applied at the periapsis and using different initial conditions, with variations in the magnitude and direction of the impulse, the energy variations due to the gravitational part of the maneuver can be changed, which means that optimal situations can be found. It happens because the orbit changes instantly when the impulse is applied, so its geometry of approach is different, including a new periapsis distance and a new angle of approach, which not only change the results of the impulsive part of the maneuver, but also the effects of the gravitational contribution of the maneuver.

Results were obtained based in the two-body and in the restricted three-body problem models, using the same variables and initial conditions as Broucke (1988), namely, the angle of approach $(\psi)$ equals to 90 degrees (condition of maximum loss of energy) and 270 degrees (condition of maximum gain of energy). The results showed that the maximum transfer of velocity and energy to the spacecraft occurs around $\alpha=-20$ degrees, where $\alpha$ is the angle between the impulse and the velocity of the spacecraft, while the minimum occurs for extreme values of $\alpha$ (around \pm 180 ) degrees. This occurs for the cases of gains of energy, considering $\psi=270$ degrees. For losses of energy $(\psi=90)$ degrees the maximum transfer is around $\alpha=20$ degrees. A comparison between the models (two and restricted three body problems) showed that the errors are smaller than $10 \%$.

A more detailed study of the powered swing-by maneuver with the impulse applied at the periapsis of the orbit of the spacecraft around the Moon was later made by (Ferreira et al. 2015), presenting a study of the maximum gains and losses of energy. Also shown in more detail were the situations where there are captures and collisions of the spacecraft with the Moon, a point that analytical approximations are not able to make. In addition to the analysis of the results, empirical equations were obtained that give the maximum and minimum variations of energy, as well as an analysis of the effect of the periapsis distance in the maneuver.

Another version of this maneuver can be made by giving freedom to the application point of the impulse. Casalino et al. (1999a) showed this more flexible maneuver, where a constraint on the periapsis altitude replaces the fixed point where the impulse is applied, so leaving the location of the application point of the impulse as a free parameter. They modeled the problem using the patched-conics approach and showed that the gains or losses of energy have some peculiarities and need to be addressed with some differences in the approach used to solve the problem. The main conclusion is that it is possible to get some extra energy by making an optimized choice of this parameter.

Following this paper, Ferreira et al. (2017) and Silva et al. (2013) made numerical mappings of the energy gains, also considering the position of the application of the impulse as a free parameter, which is used to control the maneuver. Having the magnitude and direction of the impulse as variables, this study extended the research on this problem by analyzing different positions within the sphere of influence of the secondary body to apply the impulse. This new variable of control is an angle $\theta$, measured from the periapsis line, and it defines the position for the application of the impulse. This variable must be selected such that the application point of the impulse is always inside the sphere of influence of the secondary body. As examples, different initial conditions were considered for the Earth-Moon and Sun-Jupiter systems. A superficial look at this problem suggests that the best place to apply the impulse is when the spacecraft passes by the periapsis of its orbit, because it is the point of maximum velocity, which increases the energy transfer from the impulsive maneuver to the spacecraft. However, these researches showed that, for the circular case, this is not true, and some deviations in the direction of the 
impulse and in the point of the application of the impulse can increase the energy gains of the combined maneuver.

As a natural sequence of those researches, the problem of powered swing-by was extended to systems where the primaries are in elliptical orbits (Prado 1997; Ferreira et al. 2017, 2018a,b; Qi \& Ruiter 2018a,b). This is interesting from the academic point of view, to learn the effects of the eccentricity of the primaries in this maneuver, as well as from the engineering side, because there are several systems with considerable eccentricity in the Solar System. Good examples are Mars, which has an eccentricity of 0.093, and Mercury, with an eccentricity of 0.2056 . Both of those planets have been considered many times for swing-by maneuvers (McNutt et al. 2004, 2006; Grard 2006; NASA 1999-2012, 2021; Dunne \& Burgess 1978; Jehn et al. 2008; Hollister \& Prussing 1966).

The physical reasons for the better results in terms of larger variations of energy in elliptical systems are the well-known facts that the velocity of a celestial body in an elliptic orbit is not constant, and that the larger the eccentricity, the smaller the distance between the primary bodies at the periapsis, for a fixed semi-major axis. This means that there will be an important influence of the eccentricity of the orbits on the results, considering that the energy of the spacecraft (with respect to the main body) before and after the maneuver is proportional to those parameters.

Another study of this type of maneuver was made by Qi \& Ruiter (2018b), where the optimal twoimpulsive strategy for the powered swing-by maneuver was investigated and compared with the oneimpulse case. They considered the ERTBP, with both the location and direction of the impulsive maneuver as free parameters. Numerical results showed that the swing-by epoch and the phase angle of the periapsis influence only the value of the energy variation. Other parameters influence also the capture and collision regions. For example, the increase of the magnitude of the impulsive maneuver extends these regions. However, the effect of the eccentricity of the incoming orbit is opposite to that of the impulsive maneuver. The increase of the periapsis distance enlarges the capture regions and shrinks the collision regions.

One problem that appears in this type of maneuver is the existence of errors in the magnitude and direction of the applied impulse. This problem is addressed in Ferreira et al. (2019); they show under which conditions the maneuver is still efficient, even in the presence of those errors. The literature also shows that it is possible to combine a close approach with impulsive and low thrust maneuvers (Gao et al. 2019) for a mission to the Earth's Trojan asteroid 2010TK7, which was defined as the main target for a mission that has the goal of passing by several asteroids. The multiple flyby sequence mission for asteroid explorations was proposed, and the orbit of the spacecraft was optimized for that purpose. Lowthrust and impulsive maneuvers were combined, respectively, to design the trajectories for this mission. The main goal was to reduce the fuel consumption.

Another form of powered swing-by maneuver is the one where a tether is involved. Yamasaki (2018) considers the situation where two spacecraft are linked to each other by a tether. When the tether is cut, one satellite accelerates, while the other one decelerates. This is equivalent to a propulsive maneuver that does not require additional propellant consumption, and benefits from the combination of a close approach and an impulsive maneuver.

\section{CAPTURES RESULTING FROM THE MANEUVER}

From the studies of the swing-by maneuver, it is well known that one of the consequences of its use is the capture or collision of the spacecraft by the secondary body. The impulsive maneuver can increase those occurrences, because the spacecraft can be directed to the body close enough to be captured or to collide with its surface. Some specific characteristics of the geometry generate these results, which basically come from the reduction of the energy that causes the spacecraft to slow down and, consequently, the orbital curvature to increases and to tend to approach the body.

According to Broucke (1988), the selection of the periapsis to be located such that $\psi=90$ degrees with respect to the secondary body favors the use of the gravity effect in the reduction of the energy of the spacecraft, since this geometry causes the spacecraft to pass in front of the body, and so it is decelerated due to the gravity of $M_{2}$. It is shown that captures and collisions occur, usually, for extreme values of $\alpha$, when the spacecraft is stopped and sent towards $M_{2}$ (Ferreira et al. 2017).

Still related to captures, a flyby approach and capture method for uncontrolled spacecraft capture was developed (Matsumoto et al. 2003). The basic ideas come from the gravity-assisted maneuver and the bi-elliptic transfer orbit. The results show that a smaller time of flight results in smaller savings in energy. Swing-by in the Galilean satellites 
to capture a spacecraft into the orbit of Jupiter by combining gravity assisted maneuvers and solar electric propulsion was also analyzed in the literature (Landau et al. 2010), as well as a method for capturing a Near-Earth-Asteroid (NEA) using a lunar flyby to reduce the energy required by the capture (Gong \& Li 2015). Planetary captures were considered in terms of astronautical activities (MacDonald \& McInnes 2005), as well as astronomy (Agnor \& Hamilton 2006; Nesvorny et al. 2007). They used gravity assists to understand captures of natural bodies, like moons and asteroids.

Very recent applications of powered swing-by maneuvers can be also seen in Yang et al. (2019) and Pan \& Hou (2020). Yang et al. (2019) develop a powered gravity-assist maneuver based on the pseudostate theory. Using this tool, it is possible to solve the gravity-assist segments using the restricted three-body problem to model the dynamical system. Pan \& Hou (2020) study transfers from orbits around the co-linear equilibrium point $L_{2}$ to orbits around the equilibrium triangular point $L_{4}$, considering the Earth-Moon system. They start by using the simplified circular planar restricted three body problem model for the Earth-Moon system. Two types of lowenergy transfer orbits are considered. Type-I uses planar Lyapunov orbits and powered lunar gravity assist, while type-II uses two tangential maneuvers, the first one at the departure and the second one at the nominal orbit.

\section{CONCLUSIONS}

This paper made a survey of the swing-by maneuver, showing many options available for this maneuver, as well as citing articles available in the literature on the subject. The main focus was to show more recent versions of this maneuver, which appeared in the literature after the well-known pure gravity maneuver. Initially, a historical and mathematical review of the pure gravity maneuver was made, as well as the exposition of a large list of real applications of the swing-by maneuver in space missions. The survey then explored the context of the extensions of this type of maneuver. Particular attention was given to the powered maneuver. However, different types of the propulsion studied were also presented, such as atmospheric drag, tethers, continuous propulsion, among others.

The powered swing-by combines the gravity of the body with the application of an impulse on the spacecraft, whose position of application in the orbit can vary. The best choice for the geometry can give larger energy variations to the spacecraft and, consequently, a larger velocity after it leaves the system, or can simply change the orbit within the same system. If the objective is to remove energy to insert the spacecraft in orbit around a celestial body, this type of maneuver is also efficient. The results of the various works presented showed the best configuration of the geometry and parameters of the initial orbit, in addition to the maneuver efficiency, the effect of possible errors in the assignment of parameters, the several models available to study this problem, like the patched-conics for analytical approximation and the restricted three-body problem models, in the circular and elliptic versions, for numerical studies. The text also highlighted the importance of the powered maneuver, because this is a good option when the energy obtained from the pure gravity swing-by maneuver is not enough to complete a given mission. It provides new options to the mission design, and can make possible missions that could not be achieved without this technique.

In the last section, this work presented a historical note of works that focused on the study of captures of the spacecraft occurring due to the swing-by maneuver, and described the favorable geometry for this type of event.

Finally, this survey aimed to gather a large amount of different studies on the swing-by maneuver and its extensions available in the literature. Several key results were shown, as well as mathematical and geometrical descriptions obtained from these works.

The authors wish to express their appreciation for the support provided by Grants 305210/2018-1 from the National Council for Scientific and Technological Development (CNPq); Grants 2016/24561-0, 2016/23542-1 from São Paulo Research Foundation (FAPESP) and the financial support from the Coordination for the Improvement of Higher Education Personnel (CAPES).

\section{REFERENCES}

Agnor, C. B. \& Hamilton, D. P. 2006, Natur, 441, 192, DOI: $10.1038 /$ nature04792

Anderson, R. L. 2005, Low thrust trajectory design for resonant flybys and captures using invariant manifolds (Ph.D. Thesis, University of Colorado at Boulder)

Anderson, R. L. \& Lo, M. W. 2010, JGCD, 33, 1899, DOI: $10.2514 / 1.45060$

2011, JAnSc, 58, 167, DOI: $10.1007 /$ BF03321164 
Armellin, R., Lavagna, M., \& Ercoli-Finzi, A. 2006, CeMDA, 95, 391, DOI: 10.1007/s10569-0006-9024-y

Armellin, R., Lavagna, M., Starkey, R. P., \& Lewis, M. J. 2007, JSpRo, 44, 1051, DOI: 10.2514/1.28713

Bate, R. R., Mueller, D. D., \& White, J. E. 1971, Fundamentals of astrodynamics (New York, N. Y.: Dover)

Belbruno, E. 1987, AIAA, 87

1992, AIAA, 90

1994, JBIS, 47, 73

Belbruno, E. A. \& Miller, J. K. S. 1993, JGCD, 16, 770, DOI: $10.2514-3.21079$

Belbruno, E., Topputo, F., \& Gidea, M. 2008, AdSpR, 42, 1330, DOI: 10.1016/j.asr.2008.01.018

Bonfiglio, E. P., Longuski, J. M., \& Vinh, N. X. 2000, JSpRo, 37, 768, DOI: 10.2514/2.3649

Broucke, R. A. 1988, AIAA/AAS Astrodynamics Conference, (Minneapolis, MN) 88

Byrnes, D. V. \& D'Amario, L. A. 1982, AIAA/AAS Astrodynamics Conference, (San Diego, CA) 82

Campagnola, S. \& Russell, R. P. 2010a, JGCD, 33, 463, DOI: $10.2514 / 1.44258$ . 2010b, JGCD, 33, 476, DOI: 10.2514/1.44290

Campgnola, S., Skerrit, P., \& Russel, R. P. 2012, CeMDA, 113, 343, DOI: 10.1007/s10569-012-9427-x

Carusi, A., Kresák, L., \& Valsecchi, G. B. 1995, EM\&P, 68, 71, DOI: 10.1007/BF00671499

Carusi, A., Valsecchi, G. B., \& Greenberg, R. 1990, CeMDA, 49, 111, DOI: 10.1007/BF00050709

Carusi, A., Kresák, L., \& Valsecchi, G. B. 1982, BAICz, 33,141

Carvalho, F. D. C., Fernandes, S. S., \& de Moraes, R. V. 2016, Comp. \& Appl. Math, 35, 907, DOI: 10.1007/s40314-016-0325-9

Casalino, L. \& Colasurdo, G. 2007, JGCD, 30, 1504, DOI: $10.2514 / 1.28694$

Casalino, L., Colasurdo, G., \& Pastrone, D. 1999a, JGCD, 22, 156, DOI: $10.2514 / 2.4362$ 1999b, JGCD, 22, 637, DOI: 10.2514/2.4451

Cline, J. K. 1979, CeMec, 19, 405, DOI: $10.1007 / \mathrm{BF} 01231017$

D'Amario, L. A., Byrnes, D. V., \& Stanford, R. H. 1981, JGCD, 4, 591, DOI: 10.2514/3.56115 1982, JGCD, 5, 465, DOI: 10.2514/3.56194

Deerwester, J. M. 1966, JSpRo, 3, 1564, DOI: $10.2514 / 3.28707$

Diehl, R. \& Myers, M. R. 1987, Gravity-Assist Trajectories to the Outer Solar System JPL, D-4677

Diehl, R., Belbruno, E., Bender, D., et al. 1987, AAS/AIAA Astrodynamics Conference, 65-695

Dowling, R. L., Kosmann, W. J., Minovitch, M. A., \& Ridenoure, R. W. 1990, 41th Congress of the IAF (Dresden, GDR, 6-12 Oct) 6-12 Oct.) 41th Congress of the IAF (Dresden, GDR,

Dunham, D. \& Davis, S. 1985, JAnSc, 33, 275, DOI: 10.2514/6.1984-1978

Dunne, J. A. \& Burgess, E. 1978, NASSP, 424

Edelbaum, T. N. 1959, ARS Journal, 29, 864
Elices, T. 1995, JSpRo, 32, 921, DOI: 10.2514/3.26707

Farquhar, R. W. \& Dunham, D. W. 1981, JGC, 4, 192

Farquhar, R. W., Muhonen, D., \& Church, L. C. 1985, JAnSc, 33, 235

Fernandes, S. S., Carvalho, F. D. C., \& Romao, J. V. B. 2018, RMxAA, 54, 111

Ferreira, A. F. S., Prado, A. F. B. A., \& Winter, O. C. 2015, AdSpR, 56, 252, DOI: 10.1016/j.asr.2015.04.016 . 2017, Nonli. Dyn., 89, 791, DOI: 10.1007/s11071-017-3485-2

Ferreira, A. F. S., Moraes, R. V., Prado, A. F. B. A., \& Winter, O. C. 2019, JGCD, 42, 2246, DOI: 10.2514/1.G004187

Ferreira, A. F. S., Prado, A. F. B. A., Winter, O. C., \& Santos, D. P. S. 2017, AdSpR, 59, 2071, DOI: 10.1016/j.asr.2017.01.033

2018a, Ap\&SS, 363, 145, DOI: $10.1007 /$ s10509-018-3362-6

2018b, Ap\&SS, 363, 24, DOI: $10.1007 / \mathrm{s} 10509-017-3242-5$

Flandro, G. 1966, AsAc, 12, 329, available in http:// www.gravityassist.com/IAF2/Ref.\%202-123.pdf

Formiga, J. K., Gomes, V. M., \& Moraes, R. V. 2018, Comp. \& Ap. Math., 33, 371, DOI: 10.1007/s40314017-0443-z

Gagg Filho, L. A. \& Fernandes, S. S. 2018, AcAau, 151, 228, DOI: 10.1016/j.actaastro.2018.06.006

Gao, Y., Lu, X., Peng, Y., Xu, B., \& Zhao, T. 2019, AdSpR, 63, 432, DOI: 10.1016/j.asr.2018.08.047

Gillespie, R. W. \& Ross, S. 1967, JSpRo, 4, 170, DOI: $10.2514 / 3.28830$

Gomes, V. M. \& Prado, A. F. B. A. 2008, WSEAS Transactions on Applied and Theoretical Mechanics, 3, 869 . 2010, WSEAS Trans Math., 9, 811

Gomes, V. M., Prado, A. F. B. A., \& Golebiewska, J. 2013, Sci World J., 1, DOI: 10.1155/2013/489645

Gomes, V. M., Formiga, J. K. S., \& Moraes, R. V. 2013, Math. Prob. Eng., 1, DOI: 10.1155/2013/468624

Gomes, V. M., Piñeros, J. O. M., Prado, A. F. B. A., \& Golebiewska, J. 2016, Comp. \& Ap. Math., 35, 817, DOI: $10.1007 / \mathrm{s} 40314-015-0256-\mathrm{x}$

Gong, S. \& Li, J. 2015, AdSpR, 56, 848, DOI: 10.1016/j.asr.2015.05.020

Grard, R. 2006, AdSpR, 38, 563, DOI: 10.1016/j.asr.2006.06.015

Greenberg, R., Carusi, A., \& Valsecchi, G. B. 1988, Icar, 75, 1, DOI: 10.1016/0019-1035(88)90125-X

Helton, M. R. 1972, Gravitational Capture Characteristics of the Jupiter Ganymede System (Master Thesis, UCLA)

Hiller, H. 1969, JBIS, 22, 60

Hoelker, R. F. \& Silber, R. 1959, The bi-elliptic transfer between circular coplanar orbits, Alabama: Army Ballistic Missile Agency Redstone Arsenal, (DA Tech Memo 2-59)

Hohmann, W. 1925, Die Erreichbarkeit der Himmelskörper (München und Berlin: Druck und Verlag 
R. Oldenburg)

Hollenbeck, G. R. 1975, AAS/AIAA Astrodynamics Conference on the Exploration of the Outer Planets

Hollister, W. M. \& Prussing, J. E. 1966, AsAc, 12, 169

Howell, K. C., Marchand, B. G., \& Lo, M. W. 2001, JAnSc, 49, 539, DOI: 10.1007/BF03546223

Jehn, R., Companys, V., Corral, C., García Yárnoz, D., \& Sánchez, N. 2008, AdSpR, 42, 1364, DOI: 10.1016/j.asr.2008.01.011

Jerg, S., Junge, O., \& Ross, S. D. 2009, CNSNS, 14, 4168, DOI: $10.1016 /$ j.cnsns.2008.12.009

Jezewski, D. J. \& Rozendaal, H. L. 1968, AIAAJ, 6, 2160, DOI: $10.2514 / 3.4949$

Jin, H. \& Melton, R. G. 1991, AAS/AIAA Spaceflight Mechanics Meeting, 91

Kaplan, M. H. 1976, Modern Spacecraft Dynamics \& Control (New York, NY: John Wiley \& Sons)

Kloster, K. W., Petropoulos, A. E., \& Longuski, J. M. 2011, AcAau, 68, 931, DOI: 10.1016/j.actaastro.2010.08.041

Konstantinov, M. S. \& Thein, M. 2017, AcAau, 136, 297, DOI: $10.1016 / \mathrm{j}$.actaastro.2017.02.018

Koon, W. S., Lo, M. W., Marsden, J. E., \& Ross, S. D. 2002, Celestial Mechanics: Dedicated to Donald Saari for His 60th Birthday, ed. A. Chencier, R. Cushman, C. Robinson, \& Z. J. Xia (Euanston, IL: Northwestern University), DOI: 10.1090/conm/292/04919

Koon, W. S., Lo, M. W., Marsden, J. E., \& Ross, S. D. 2000, Chaos, 10, 427, DOI: 10.1063/1.166509

. 2001, CeMDA, 81, 63, DOI: 10.1023/A:1013359120468

2008, Dynamical Systems, the Three-Body Problem and Space Mission Design (Marsden Books)

Landau, D., Strange, N., \& Lam, T. 2010, AAS/AIAA Spaceflight Mechanics Conference, Paper AAS 10-169

Lavagna, M., Povoleri, A., \& Finzi, A. E. 2005, AcAau, 57, 498, DOI: 10.1016/j.actaastro.2005.03.052

Lawden, D. F. 1953, ARS Journal, 23, 360, DOI: $10.2514 / 8.4642$ 1954, JBIS, 13, 87

Lewis, M. J. \& McRonald, A. D. 1992, JSpRo, 29, 653, DOI: $10.2514 / 3.11506$

Lohar, F. A., Misra, A. K., \& Mateescu, D. 1996, AcAau, 38, 445, DOI: 10.1016/0094-5765(96)00017-3

Lohar, F. A., Mateescu, D., \& Misra, A. K. 1994, AcAau, 32, 89, DOI: 10.1016/0094-5765(94)90059-0 1997, JSpRo, 34, 16, DOI: $10.2514 / 2.3186$

Longman, R. W. \& Schneider, A. M. 1970, JSpRo, 7, 570, DOI: $10.2514 / 3.29992$

MacDonald, M. \& McInnes, C. 2005, JGCD, 28, 365, DOI: $10.2514 / 1.11866$

Machuy, A. L., Prado A. F. B. A., \& Stuchi, T. J. 2007, AdSpR, 40, 118, DOI: 10.1016/j.asr.2007.02.069

Marsh, S. M. \& Howell, K. C. 1988, Double Lunar Swing by Trajectory Design, AIAA paper $88-4289$

Matsumoto, S., Dubowsky, S., Jacobsen, S., \& Ohkami, Y. 2003, AIAA Guidance, Navigation, and Control Conference, 5745, DOI: https://doi.org/10.2514/6.2003-5745

McConaghy, T., Debban, T. J., Petropulos, A. E., \& Longuski, J. M. 2003, JSpRo, 40, 380, DOI: $10.2514 / 2.3973$

McNutt Jr., R. L., Solomon, S. C., Gold, R. E., \& Leary, J. C. 2006, AdSpR, 38, 564, DOI: 10.1016/j.asr.2005.05.044

McNutt Jr., R. L., Solomon, S. C., Grard, R., Novara, M., \& Mukai, T. 2004, AdSpR, 33, 2126, DOI: 10.1016/S0273-1177(03)00439-3

McRonald, A. D. \& Randolph, J. E. 1992, JSpRo, 29, 216, DOI: $10.2514 / 3.26337$

Minovitch, M. A. 1961, Pasadena: JPL, 47 (JPL Tec. Memo 312-130, Aug. 23)

Murcia, J. O. P., Prado, A. F. B. A., \& Gomes, V. M. 2018a, RMxAA, 54, 143 2018b, RMxAA, 54, 485

Murray, C. D. \& Dermott, S. F. 2000, Solar System Dynamics (Cambridge, UK: CUP), DOI: https://doi. org/10.1017/CB09781139174817

NASA 1999-2012, "Messenger Trajectory Developed by JHU/APL", available at http://messenger.jhuapl. $\mathrm{edu} /$ themission

NASA 2021, "Nasa Space Science Data Coordinate Archive: Mariner 10", available at: https://nssdc.gsfc. nasa.gov/nmc/spacecraftDisplay.do?id=1973-085A

Negri, R. B. \& Prado, A. F. B. A. 2020, Journal of the Brazilian Society of Mechanical Sciences and Engineering, 42, 406, DOI: https://doi.org/10.1007/ s40430-020-02489-x

Negri, R. B., Prado, A. F. B. A., \& Sukhanov, A. 2017, CeMDA, 129, 269, DOI: 10.1007/s10569-017-9779-3

Negri, R. B., Sukhanov, A., \& Prado, A. F. B. A. 2019, AdSpR, 64, 42, DOI: 10.1016/j.asr.2019.04.002

Nesvorný, D., Vokrouhlický, D. \& Morbidelli, A. 2007, AJ, 133, 1962

Nock, K. T. \& Upholf, C. W. 1979, AAS/AIAA paper 79-165

Okutsu, M., Yam, C. H., \& Longuski, J. M. 2016, AIAA Paper 2006-6745

Öpik, E. J. 1976, Interplanetary Encounters: Close-range gravitational interactions (Amsterdam: Elsevier Scientific Publishing)

Ozimek, M. T. \& Howell, K. C. 2010, JGCD, 33, 533, DOI: $10.2514 / 1.43179$

Pan, S. S. \& Hou, X. Y. 2020, AdSpR, 65, 2878, DOI: 10.1016/j.asr.2020.03.022

Piñeros, J. O. M. \& Prado, A. F. B. A. 2017, Ap\&SS, 362, 120, DOI: $10.1007 /$ s10509-017-3097-9

Pourtakdoust, S. H. \& Sayanjali, M. 2014, Nonlinear Dyn., 76, 955

Prado, A. F. B. A. 1996, JGCD, 19, 1142, DOI: $10.2514 / 3.21756$

1997, JGCD, 20, 797, DOI: 10.2514/2.4115

Prado, A.F.B.A. \& Broucke, R. 1995, AcAau, 36, 285, DOI: 10.1016/0094-5765(95)00114-X

Prussing, J. E. 1992, JGCD, 15, 1037, DOI: $10.2514 / 3.20941$ 
Qi, Y. \& Ruiter, A. 2018a, MNRAS, 478, 1392, DOI: $10.1093 / \mathrm{mnras} / \mathrm{sty} 1155$ 2018b, MNRAS, 481, 4621

2020, JGCD, 43, 111, DOI: 10.2514/1.G004358

Qi, Y., Xu, S., \& Qi, R. A. 2017, MNRAS, 468, 2265, DOI: $10.1093 / \mathrm{mnras} / \mathrm{stx} 505$

Qian, Y. J., Zhang, W., Yang, X. D., \& Yao, M. H. 2016, Nonlinear Dyn., 85, 463

Randolph, J. E. \& McRonald, A. D. 1992, JSpRo, 29, 223, DOI: $10.2514 / 3.26338$

Ross, S. D. \& Scheeres, D. J. 2007, SJADS, 6, 576, DOI: $10.1137 / 060663374$

Roth, H. L. 1965, No. Aerospace corp. el. Segundo ca. el. Segundo technical operations (TDR-469 (5540-10)-5)

Ruppe, H. O. 1967, Introduction to Astronautics, Vol. 2 (New York, NY: Academic Press)

Shternfeld, A. 1959, Soviet Space Science: The Russian Story of Artificial Satellites (New York, NY: Basic Books Inc)

Silva, A. F., Prado, A. F. B. A., \& Winter, O. C. 2013b, JPhCS, 465, 012001, DOI:10.1088/1742$6596 / 465 / 1 / 012001$

Sims, J. A., Longuski, J. M., \& Patel, M. R. 1995, AcAau, 35, 297, DOI: 10.1016/0094-5765(94)00195-R 2000, JSpRo, 37, 49, DOI: 10.2514/2.3525

Strange, N. J. \& Longuski, J. M. 2002, JSpRo, 39, 9, DOI: $10.2514 / 2.3800$

Striepe, S. A. \& Braun, R. D. 1991, J. Astronaut. Sci., 39,299
Sukhanov, A. A. 1999, AcAau, 45, 177, DOI: 10.1016/S0094-5765(99)00135-6

Sukhanov, A. A. \& Prado, A. F. B. A. 2001, JGCD, 24, 723, DOI: $10.2514 / 2.4772$

Szebehely, V. 1967, Theory of Orbits. The restricted problem of three bodies (New York, NY: Academic Press)

Szebehely, V. \& Giacaglia, G. E. O. 1964, AJ, 69, 230, DOI: $10.1086 / 109261$

Valtonen, M. \& Karttunen, H. 2006, The Three-Body Problem (Cambridge, UK: CUP)

Vasile, M. \& Campagnola, S. 2009, JBIS, 62, 15

Woolley, R. C. \& Scheeres, D. J. 2009, Advances in the Astronautical Sciences, 135, 1245

Yamakawa, H. 1992, On Earth-Moon Transfer Trajectory with Gravitational Capture (PhD. Thesis, University of Tokyo)

Yamakawa, H., Kawaguchi, J., Ishii, N., \& Matsuo, H. A. 1992, AAS/AIAA Spaceflight Mechanics Meeting, AAS Paper 92-186 1993, AAS/AIAA Astrodynamics Specialist Conference, AAS Paper 93-633

Yamasaki, T., Bando, M., \& Hokamoto, S. 2018, AcAau, 142, 212, DOI: 10.1016/j.actaastro.2017.11.002

Yang, B., Yang, H., \& Li, S. 2019, AcA, 165, 139

Zhang, Ch., Topputo, F., Bernelli-Zazzera, F., \& Zhao, Y. 2015, JGCD, 38, 1501, DOI: 10.2514/1.G001080

Zotos, E. E. 2015, Nonlinear Dynamics, 82, 1233, DOI: $10.1007 / \mathrm{s} 11071-015-2229-4$

Rodolpho V. de Moraes: National Institute for Space Research - INPE, CEP 12227-010, São José dos Campos - SP, Brazil (rodolpho.vilhena@gmail.com).

Alessandra F. S. Ferreira: Department of Mathematics - São Paulo State University (FEG-UNESP), CEP 12516410, Campus of Guaratinguetá - SP, Brazil (alessandra.ferraz@unesp.br).

Antonio F. B. A. Prado: Volunteer Professor at Peoples' Friendship University of Russia (RUDN University), CEP 117198, Moscow, Russian Federation (antonio.prado@inpe.br). 